\title{
Economía urbana y dispersión metropolitana: el rol de las cadenas de supermercado en la producción del espacio metropolitano en Belém (Pará, Brasil)
}

\author{
Economia urbana e dispersão metropolitana: o papel das cadeias de supermercado \\ na produção do espaço metropolitano em Belém (Pará, Brasil) \\ Urban economy and metropolitan dispersion: the role of supermarket chains in the \\ production of the metropolitan space in Belém (Pará, Brazil)
}

AUTOR

Tiago Veloso dos Santos*

tiago.veloso@ifpa. edu.br

* Doctor en Desarrollo Sostenible por el Núcleo de Altos Estudos Amazônicos da Universidade Federal do Pará (NAEA, UFPA, Brasil). Docente del Instituto Federal de Educação, Ciência e Tecnologia do Pará (IFPA, Brasil).

\section{RESUMEN:}

En el contexto de la dispersión de la Región Metropolitana de Belém, el presente trabajo analiza la actuación de uno de los agentes productores de espacio urbano con mayor influencia en la economía urbana de la metrópolis: las cadenas de supermercados minoristas. La investigación se centra en la lógica de actuación del grupo "líder" y su relación con la tendencia a la dispersión del tejido metropolitano, encabezado por la ciudad Belém. Se parte de la premisa de la emergencia de los grupos de supermercados minoristas como manifestación de una economía metropolitana organizada en torno al sector terciario moderno, especialmente al comercio y los servicios. La metodología de este trabajo abarca tres momentos: revisión bibliográfica, revisión documental, observaciones sistemáticas de campo y producción cartográfica de los procesos observados y datos estadísticos obtenidos.

\section{RESUMO:}

No contexto de dispersão da Região Metropolitana de Belém, analisa-se a atuação de um dos agentes produtores de espaço urbano com maior atuação na economia urbana da metrópole, as redes de supermercado varejistas. Trata-se, portanto, de analisar as lógicas de atuação da rede de supermercados "Líder" e a relação com a tendência de espraiamento da malha metropolitana nucleada por Belém. Parte-se da premissa da emergência dos grupos de supermercado varejista como expressão de uma economia metropolitana organizada em torno do setor terciário moderno, especialmente comércio e serviços. O percurso metodológico está compreendido em três momentos: levantamento bibliográfico, levantamento documental, observações sistemáticas de campo com produção de cartografia dos processos observados e dados estatísticos levantados.

\footnotetext{
ABSTRACT:

In the context of the dispersion of the Metropolitan Region of Belém, the performance of one of the agents producing urban space with greater performance in the urban economy of the metropolis, the retail supermarket chains, is analyzed. Therefore, it is about analyzing the logics of the "Líder" supermarket chain and the relationship with the spreading trend of the metropolitan network nucleated by Belém. It starts from the premise of the emergence of retail supermarket groups as an expression of a metropolitan economy organized around the modern tertiary sector, especially commerce and services. The methodological path of the research is understood in three moments: bibliographic survey, documentary survey, systematic field observations with later production of cartography of the observed processes and statistical data collected.
} 


\section{Introducción}

La urbanización social y el territorio brasileño se han expandido y en su geografía reciente se distingue con una importante regularidad la diseminación de aglomerados metropolitanos como manifestación territorial de lo que Lencioni (2008) denomina metropolización del espacio ${ }^{1}$, una forma-producto espacial de las dinámicas del capitalismo contemporáneo, de las tendencias incrementadas de concentración y centralización del capital en los espacios urbanos con más densidad. En el caso brasileño, los procesos de metropolización del espacio son, aparentemente, la repercusión más intensa de la urbanización del territorio² (Santos \& Silveira, 2001).

Estos procesos son característicos de la urbanización contemporánea. Así, su propagación por el territorio nacional, junto a los procesos actuales de metropolización del espacio, no se rigen por un patrón homogéneo. En cambio, existe una variedad de formas metropolitanas que resultan de distintos procesos de producción de los espacios en las regiones del territorio nacional.

En ese caso específicamente, desde la realidad urbana de la región amazónica, se toma como referencia empírica de análisis la Región Metropolitana de Belém (RMB), segunda principal aglomeración metropolitana de la Amazonia brasileña ${ }^{3}$. La región en cuestión ha presentado una marcada alteración de su paisaje natural de bosque tropical, debido a las transformaciones provocadas por las formas de producción del espacio regional desde mediados del siglo XX, que sitúa a las ciudades y lo urbano como principal referencia territorial a principios del siglo XXI.

Asumiendo este cambio como una característica de la urbanización contemporánea, es pertinente realizar un análisis del método que la originó, y, además, comprender los agentes constitutivos que generan esa forma urbana: la metrópolis, considerada no solo como la simple unidad físico-territorial de la mancha urbana, y sí como la concentración y propagación de flujos de materiales diversos que son producto, condición y medio de la producción del espacio, cada vez más prolijos en lo que a relaciones socio-espaciales se refiere.

El reconocimiento de dichos cambios es posible a través del análisis de las acciones materializadas en la metrópolis por los agentes productores del espacio urbano, identificados por Corrêa (1989) como el Estado, los propietarios de los medios de producción, los promotores inmobiliarios y los grupos sociales excluidos. Se parte de la premisa de que la acción orientada de esos agentes se guía por una lógica capitalista hegemónica como infraestructura económica, que impulsa la producción y reproducción del espacio urbano en tanto instancia social.

En este caso, tratándose del espacio metropolitano de Belém, distinguimos la relación de un conjunto de emprendimientos comerciales realizados por cadenas de supermercados importantes a escala urbana y regional. Dichos emprendimientos son una manifestación de la relevancia del sector terciario moderno en la producción del espacio metropolitano, a diferencia de otras realidades urbanas de Brasil, en las que el capital industrial es el principal vector de inducción de la expansión de la red urbana. Así, esos emprendimientos están ubicados estratégicamente en determinados locales.

La expansión de las cadenas de supermercados, en ese aspecto, es una manifestación de procesos más profundos de metropolización del espacio en Belém, en la cual existe un aumento fundamental de los flujos intermunicipales, la producción de asentamientos habitacionales para los estratos más pobres, así como también los producidos para estratos medios y altos, situándose cada vez más lejos del núcleo metropolitano; además de la instalación de grandes objetos para posibilitar la fluidez en tal espacio. 
Reconociendo lo singular de esta realidad metropolitana y regional en el territorio brasileño, se parte de la hipótesis de la existencia de una relación entre la producción del espacio metropolitano en Belém y los métodos de expansión empresarial de las cadenas de supermercados y las reformas materiales facilitadas por la ubicación territorial de esos emprendimientos. Se entiende que, refiriéndose a una metrópolis estructurada de manera fraccionada, en torno a la expansión del circuito superior moderno de la economía urbana (evidentemente por el capital comercial del sector servicios), existe una relación de interdependencia entre la dispersión del espacio metropolitano de Belém y la expansión de los emprendimientos comerciales de dichas cadenas.

Esta relación está consolidada desde la década de 1960, en el contexto de un conjunto de transformaciones sucedidas en la región. Éstas nacieron de las repercusiones del proceso de integración nacional y posibilitaron la emergencia de capitales comerciales vinculados al crecimiento de las cadenas de distribución minoristas, por lo tanto, directamente relacionadas al desarrollo de una economía urbana en la principal capital de la región amazónica en la época: Belém.

En términos teóricos, se parte de la teoría de la producción social del espacio urbano (Lefebvre, 2001) y la manera en que dicha producción está intrínsecamente vinculada a ciertos agentes sociales productores de este espacio, en particular las premisas sobre la realidad brasileña propuestas por Correa (1989).

Desde la perspectiva metodológica se llevó a cabo un estudio cualitativo, vinculando la hipótesis teórica de la emergencia de la metrópolis en Belém al auge económico de grupos y cadenas de supermercados. Para ello, como técnica de investigación, se empezó el recorrido metodológico con una revisión bibliográfica de la temática analizada: el progreso de Belém como metrópolis regional durante la segunda mitad del siglo XX junto al surgimiento del sector terciario moderno en la producción más reciente en la distribución espacial de la Región Metropolitana de Belém. Este procedimiento fundamentó la reconstitución del proceso histórico de producción del espacio metropolitano de Belém, asociándolo a la formación de una economía urbana caracterizada por la hegemonía de los grupos empresariales del sector terciario, que paulatinamente va migrando de capitales comerciales de procedencia tradicional hacia la organización alrededor del circuito superior moderno de la economía urbana.

Asimismo, en la investigación documental se buscaron contribuciones a la comprensión del rol de ese capital comercial expresado por la importancia de las cadenas de supermercados, especialmente la del Grupo Líder, referencia empírica del análisis. La revisión de la legislación específica, de los registros periodísticos, de los informes comerciales de las mismas cadenas minoristas, además de las entrevistas a los representantes de dichas cadenas concedidas a los medios de comunicación, posibilitó la caracterización de una genealogía del proceso de formación de las mismas, asociándola a la reorganización económica de la ciudad a partir de la segunda mitad del siglo XX.

Un tercer aspecto que considerar de las elecciones metodológicas, articuladas para este análisis, es que hicieron posible la identificación de agentes para la construcción de una cartografía de expansión de las cadenas de supermercados, corroborando la conexión con los procesos de dispersión metropolitana ocurridos en Belém.

Es justamente tal proceso el que será abordado en el presente trabajo, estructurado inicialmente respecto a la presentación del tipo de metropolización en Belém y su relación con la progresiva evolución del circuito superior moderno de la economía urbana, donde surge el Grupo Líder, emprendimiento analizado como muestra de la formación de una economía metropolitana en Belém con sus respectivas modificaciones económicas.

\section{El proceso de metropolización en Belém y el circuito superior de la economía urbana}

La producción del espacio metropolitano en Belém mantiene particularidades provenientes de su formación en tanto ciudad de referencia regional. El contexto geográfico reconoce la historia de la formación metropolitana de Belém vinculada a los cambios determinados a inicios de la segunda mitad del siglo XX, ya 
que, de hecho, hasta mediados de la década de 1960, la ciudad aún se encontraba reducida a un espacio relativamente próximo a su local de fundación. La periodización presentada por Moreira (1989) respecto al crecimiento urbano de Belém afirma lo siguiente:

En un principio, la ciudad se expandió acompañando el corredor fluvial, para, seguidamente, interiorizarse y continentalizarse, puntualizando las tres primeras fases de su crecimiento: la pequeña ribera (de la fundación de la ciudad en 1616 hasta mediados del siglo XVIII); la de penetración o interiorización (de mediados del siglo XVIII a mediados del siglo XIX); y la de la continentalización (de mediados del siglo XIX en adelante) (Moreira, 1989, p. 52).

De este modo, únicamente durante el último período, según Trindade Júnior (1998), se puede afirmar la configuración de una cuarta fase de expansión urbana, marcada por la dinámica de la metropolización, que supone el avance del tejido urbano con relación al período y las fases anteriores:

Añadiríamos a esa periodización de Moreira (1989), otra fase, la de metropolización, la cual se inicia en la década de los sesenta y se afianza en las siguientes décadas y que supone la incorporación de las ciudades y villas cercanas a Belém, definiendo un tejido urbano único, aunque fragmentado (Trindade Júnior, 1998, p. 3).

En ese caso, se entiende que Belém tuvo su momento de expansión orientado a la formación de un tejido metropolitano más complejo a partir del año 1960, contextualizado en la alteración de las redes de circulación, con la construcción de la autopista Belém-Brasília, primer gran eje de penetración por carretera de la Cuenca Amazónica (Vicentini, 2004), y que es un elemento relevante para comprender la metropolización regional. Esta etapa, posibilitada por la llegada de la autopista, tiene un impacto considerable en la dinámica de crecimiento poblacional de Belém como se muestra en la Tabla 1:

Tabla 1. Belém: evolución de la población (1950 - 2010)

\begin{tabular}{|l|l|}
\hline AÑO & POBLACIÓN TOTAL \\
\hline 1950 & 254949 \\
\hline 1960 & 402170 \\
\hline 1970 & 642514 \\
\hline 1980 & 949545 \\
\hline 1991 & 1.244 .688 \\
\hline 2000 & 1.279 .861 \\
\hline 2010 & 1.393 .399 \\
\hline
\end{tabular}

Fuente: Instituto Brasileiro de Geografia e Estatística (IBGE, 2010).

Dispuesto así el inicio de su estructura metropolitana, es posible comprender la metropolización regional a partir del desarrollo de la infraestructura que viabilizó el proyecto de integración de la Amazonia al resto de la economía nacional mediante la articulación de esas redes viales. Los cambios en el rol de la región también presentaron consecuencias en la producción del espacio urbano de Belém. El Mapa 1 muestra la tendencia de la expansión de Belém en dirección a los municipios colindantes al inicio de la década de 1960, como representación cartográfica de esos cambios en la producción del espacio urbano y la formación de una Belém metropolitana. 
Las modificaciones fisiográficas en el perfil de producción del espacio no escapan a las alteraciones en la estructura económica de la ciudad. Incluso, según Trindade Júnior (2000;2006), Belém no experimentó el estímulo de crecimiento impulsado a partir del sector industrial. Su perfil caracteriza a una ciudad situada entre las que presentan una condición metropolitana, sobresaliendo en actividades comerciales y de servicios. Sus industrias - con un peso relativamente pequeño - apuntaron al aprovechamiento de materias primas propias de la región y, en consecuencia, con un impacto relativamente mayor con relación a la selva.

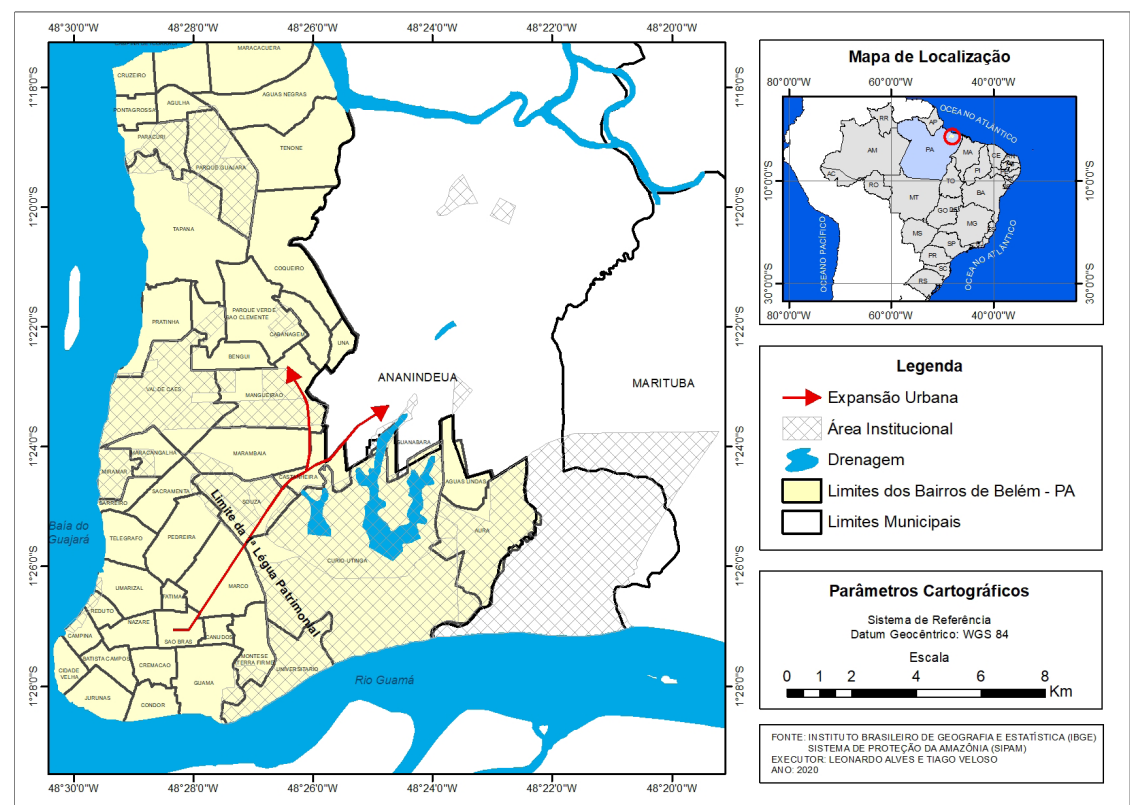

Mapa 1. Belém: expansión del espacio urbano. Fuente: Santos (2017).

La participación relativa de los sectores industriales (Tabla 2), y del sector de servicios y comercio (Tabla 3) en la Región Metropolitana en perspectiva temporal ${ }^{4}$, manifiesta la relevancia de cada uno de ellos en la producción del espacio metropolitano, así como la importancia relativa de los sectores dentro de un contexto más general:

Tabla 2. Región Metropolitana de Belém: evolución de la participación del sector industrial con relación al PIB del estado de Pará5

\begin{tabular}{|c|c|c|c|}
\hline & $\begin{array}{r}\text { METROPOLITANA } \\
\text { DE BELÉM }\end{array}$ & ESTADO DE PARÁ & RMB / ESTADO (\%) \\
\hline 1949 & $104.290,52$ & $128.797,20$ & 80,97 \\
\hline 1959 & $262.844,71$ & $342.012,58$ & 76,79 \\
\hline 1970 & $494.356,81$ & $647.642,43$ & 76,33 \\
\hline 1980 & $2.062 .517,19$ & 4.106.902,95 & 50,22 \\
\hline 1996 & $1.677 .132,96$ & $3,408.066,56$ & 48,92 \\
\hline 2000 & $2.213 .298,31$ & $4.889 .652,33$ & 45,27 \\
\hline 2010 & $2.633 .446,64$ & $13.122 .002,58$ & 20,10 \\
\hline
\end{tabular}

Fuente: IBGE (2010); Ipeadata (2019).

En efecto, fue la llegada de la autopista y los consiguientes impactos económico-espaciales de ese evento los que generaron cambios significativos en la economía urbana en Belém. Por un lado, se percibe la progresiva desarticulación de formas tradicionales de la organización económica ${ }^{6}$ y de un pequeño sector industrial ${ }^{7}$ vinculado a la producción de bienes de consumo inmediato debido al relativo aislamiento de la economía amazónica con relación al territorio brasileño. Por otra parte, ese redireccionamiento de la economía urbana abre una posibilidad de mayor organización del capital comercial respecto a su expansión y, posteriormente, de la modernización de la distribución de productos y bienes que necesitarían ser reorganizados en la nueva dinámica económica, ahora asociada al amplio proceso de integración regional al desarrollo económico nacional. 
- ECONOMÍA URBANA Y DISPERSIÓN METROPOLITANA: EL ROL DE LAS CADENAS DE SUPERMERCADO

EN LA PRODUCCIÓN DEL ESPACIO METROPOLITANO EN BELÉM (PARÁ, BRASIL)

TIAGO VELOSO DOS SANTOS

Tabla 3. Región Metropolitana de Belém: evolución de la participación del sector terciario con relación al PIB del estado de Pará

\begin{tabular}{|c|c|c|c|}
\hline & $\begin{array}{r}\text { METROPOLITANA } \\
\text { DE BELÉM }\end{array}$ & ESTADO DE PARÁ & RMB / ESTADO (\%) \\
\hline 1949 & $125.317,52$ & $162.994,40$ & 76,90 \\
\hline 1959 & $797.107,52$ & $1.064 .601,23$ & 74,97 \\
\hline 1970 & $619.179,46$ & $774.679,88$ & 79,94 \\
\hline 1980 & $1.033 .878,48$ & $1.590 .372,79$ & 65,01 \\
\hline 1996 & $1.677,132,96$ & $2.280 .157,74$ & 73,56 \\
\hline 2000 & 2. $213.298,31$ & $4.692 .473,00$ & 47,20 \\
\hline
\end{tabular}

Fuente: IBGE (2010); Ipeadata (2019)

Es ese proceso el que configura el contexto económico específico de desarrollo de un sector terciario que, a partir de allí, liderará el circuito superior moderno ${ }^{8}$ de la economía urbana respecto al periodo anterior que dependió en gran medida de la dinámica de los sectores tradicionales. La Tabla 4 muestra el surgimiento de grupos empresariales relacionados con el sistema terciario moderno, los cuales inician su trayectoria de negocios en Belém en las décadas que sucedieron a la apertura de la autopista:

Tabla 4. Belém: surgimiento de grupos empresariales durante el período (1950 - 1970)

\begin{tabular}{|c|c|c|c|}
\hline EMPRESA & LOGO & AÑO DE FUNDACIÓN & $\begin{array}{l}\text { SECTOR DE ACTUACIÓN } \\
\text { EN SU ORIGEN }\end{array}$ \\
\hline Y. Yamada & YYAMADA & 1950 & $\begin{array}{l}\text { Supermercado y tienda por } \\
\text { departamento }\end{array}$ \\
\hline Visão & 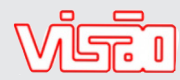 & 1965 & Tienda por departamento \\
\hline $\begin{array}{l}\text { Líder Comércio e Indústria } \\
\text { LTDA }\end{array}$ & & 1964 & Supermercado \\
\hline Grupo Formosa & & 1975 & $\begin{array}{l}\text { Supermercado y tienda por } \\
\text { departamentos }\end{array}$ \\
\hline $\begin{array}{l}\text { Nazaré Comércio de } \\
\text { Alimentação e Magazine } \\
\text { LTDA }\end{array}$ & Dazaré & 1976 & $\begin{array}{l}\text { Supermercado y tienda por } \\
\text { departamentos }\end{array}$ \\
\hline
\end{tabular}

Fuentes: canales institucionales de los grupos ([http://www.grupolideronline.com.br]; [http://www.grupolideronline.com.br]; [https://www.gruponazare.net/historia]). Fuente secundaria: Junta Comercial del estado de Pará.

De esta manera, es posible interferir en el afianzamiento de una economía urbano-metropolitana en Belém que tiene lugar a partir del surgimiento de esas cadenas de comercio minorista, debido a que se trata de un tipo de organización que conlleva procesos más profundos de circulación de mercancías; y sin limitarse a eso, percibiendo que la operación propia de esos grupos también incluye importantes intercambios de información, como parte de su respectivo proceso de gestión. 
Todos los grupos enumerados se fundaron originalmente en Belém. Aunque hayan expandido su radio de acción hacia otras regiones del Estado de Pará, o incluso hasta otros estados como es el caso particular de la empresa Y. Yamada ${ }^{9}$, operan principalmente en el espacio metropolitano de Belém, donde se encuentra la mayoría de sus tiendas. Las redes, además de haber sido fundadas en Belém, mantienen hoy en día sus sedes y centros de distribución en la capital paraense.

Una vez más, la formación de esa economía urbana, durante el período de 1950 y 1960, se relaciona con los cambios más estructurales de la economía paraense, tal como es descrito por Tupiassu y Santos (1967).

El sector primario de la economía paraense comienza a sufrir modificaciones, la agricultura se diversifica un poco, en especial debido a las técnicas introducidas por inmigrantes (...) con la aparición de la Superintendencia del Plan de Valorización Económica de la Amazonia, en 1954, el panorama mejora con la instalación de una nueva planta generadora de energía eléctrica en la capital, la cual tiene un importante efecto germinativo. La construcción civil se fue perfeccionando y expandiendo. Las alfarerías incrementan la producción de material de construcción. El comercio minorista se moderniza. (...). Crece la variedad de bienes de consumo de origen industrial, generando nuevos establecimientos en el ramo de las gaseosas, confección de ropa y muebles. Entre 1950 y 1959, se establecerán nuevas conexiones viales (...). De esa forma, la urbanización en el sentido demográfico, se precipita, dando lugar a un incremento de las comunicaciones sociales en los centros de aglomeración (Consejo Nacional de Economía, 1962, p. 270, negrita del autor).

Para Santos (2004), las grandes tiendas y los supermercados son la representación de un fenómeno que se expande en los países periféricos. La existencia de dichos emprendimientos está relacionada a la posibilidad de una demanda más numerosa y diversificada, así como a la oportunidad de transacciones en dinero efectivo o según las formas burocráticas de crédito particulares de algunas firmas comerciales.

Los cambios urbano-regionales ya resaltados se empeñan en viabilizar este contexto de extensión de una economía metropolitana que pasa, ineludiblemente, por la necesidad de modernización de la gestión de las firmas, de la ampliación de la base monetaria junto al consecuente impacto en las formas de oferta de crédito al consumo y que tendrán consecuencias en la producción del espacio. Según Santos (2004):

En este tipo de supercomercio las relaciones son impersonales. $Y$ por esto es que la cantidad de supermercados varía principalmente según la importancia de la clase media y del número de asalariados, mientras que su dimensión está en función de la densidad de población en los barrios ricos (Santos, 2004, p. 87).

Adoptado como referencia más inmediata del análisis sobre la evolución de ese sector en la economía urbana y en la transformación metropolitana de Belém, el origen de la cadena de supermercados "Líder" será el siguiente punto que abordar, relacionado a nuevas dinámicas de producción del espacio urbano y regional.

\section{Los orígenes del Grupo Líder en el contexto de la reorganización económica de Belém}

La inauguración de la autopista Belém-Brasilia, como expresión estructural de las políticas de integración nacional, suscita una drástica modificación en la economía del espacio urbano de Belém. Browder y Godfrey (2006) Ilaman la atención sobre el hecho de que es en el periodo posterior a la Segunda Guerra Mundial cuando Belém se convierte en el centro administrativo regional para programas de desarrollo en la Cuenca Amazónica, siendo esa la función principal de la ciudad en el ámbito regional desde este periodo.

Dicha función genera un impacto importante desde una perspectiva de producción del espacio urbano y metropolitano en la ciudad, considerando que es en él donde se asignan diversas instituciones para implementar el proyecto de integración en la región ${ }^{10}$. 
En el aspecto económico, el sector industrial, que precozmente existía en la ciudad, se vuelve desestructurado en virtud de la pérdida de protección que deriva de dos factores: las políticas de integración implementadas por el gobierno federal, con énfasis en la abertura de grandes autopistas; y el acceso concedido a las fuentes de materias primas y penetración del capital extranjero en diferentes actividades manufactureras (Trindade Júnior, 1997, p. 89).

La pérdida de protección como consecuencia de los impactos en la transformación de la logística de transportes de la región puede interpretarse como la pérdida de una ventaja comparativa del sector industrial regional, el cual es desestructurado a causa de la reducción de los costos de transacción resultantes de la modernización del tejido vial. De forma simultánea a la desestructuración de la incipiente industria regional, en el sector terciario de la economía urbana se producen modificaciones fundamentales, ya que a principios del año 1950 las relaciones de precio de intercambio exterior atraviesan un declive apreciable.

Desde una perspectiva teórica, ese aspecto es esencial para entender el tipo de inserción subordinada de la economía regional amazónica al parque industrial brasileño, concentrado en el eje sudeste del país, puesto que, específicamente en este caso, las modificaciones en la tecnología y transporte alteraron la ventaja comparativa de la región, incluso sin hacer posible la diversificación y el incremento técnico del parque industrial brasileño existente, pero sí integrándola de manera dependiente respecto al resto del país. En ese contexto, el desarrollo de otra lógica de transportes, la sustitución, o mejor complementación de la navegación fluvial por el tejido vial, orientó el reforzamiento de la dependencia, reduciendo y, finalmente, eliminando la ventaja comparativa.

La paulatina integración de los supermercados regionales con la industria del sudeste brasileño cambia las relaciones de intercambio, dirigiendo cada vez más las exportaciones paraenses hacia el mercado interno nacional. Sin embargo, a lo largo de la década los importadores paraenses oscilan entre el mercado externo y el interno, de modo que, en general, las importaciones del resto de Brasil de mantienen por debajo del $60 \%$ del total de tonelada importada (Tupiassu; Santos, 1967).

La distancia y la dificultad de transporte hacia Pará (los medios de transporte eran aéreos, fluviales y marítimos hasta el final del año 1950) impulsaron significativamente el flete de las mercancías originarias de otras regiones y las encarecieron. Con esto, los productos locales se resentían poco de la competencia de las importaciones (Mourão, 1989, p. 46).

Durante toda la década de 1950, por lo tanto, surge una nueva etapa en la economía paraense, resultante de una mayor integración entre la economía de Pará y el mercado interno brasileño. Las exportaciones paraenses resaltan su preferencia hacia el mercado interno brasileño.

Desde el punto de vista de mercado de trabajo se da una transformación aún más esencial, debido a que, durante ese período, por lo menos en el sector primario, era común el sistema de provisión de mercancía a crédito, en el cual el agricultor podía garantizar los productos de subsistencia en forma de préstamos al vendedor y este pagaba conforme a la producción agrícola. La sustitución progresiva de dicho sistema por el cada vez más frecuente proceso de pago de salarios actúa apuntando a la transformación de economía regional en economía monetizada.

Es en este contexto de cambios urbano-regionales que emprendimientos comerciales, como los que se muestran en la Tabla 4, se tornan importantes en la economía urbana de la ciudad. El supermercado "Líder" es un resultado directo de dichas modificaciones, puesto que inicialmente era solo un pequeño establecimiento comercial de venta de artículos de consumo básico, es decir, productos perecederos, procedente de la acumulación de capitales del comercio tradicional por medio de la red fluvial de la región. Según lo descrito por uno de sus fundadores, el empresario Oscar Rodrigues, en una entrevista:

Comprábamos la cachaza. En aquella época éramos muy astutos, Belém, Igarapé-Miri, Abaeté, eran de los mayores productores. Y aún no teníamos la autopista Belém-Brasilia, entonces se dio 
esa invación (SIC) de productos provenientes de allá. Y así, Igarapé-Miri, Abaeté (Municipio de Abaetetuba), abastecía toda la cachaza al Bajo Amazonas, Manaos (Entrevista de Oscar Rodrigues para el Programa "Papo no Tucupi" [https://www.youtube.com/watch?v=53TUSE45iNg]).

El testimonio del empresario resalta, inclusive, la existencia de un intenso comercio fluvial que hacía posible el traslado de las mercancías, y de qué manera esa red era importante para el abastecimiento del espacio urbano de Belém durante el periodo:

Estuve vendiendo cachaza de botellón en canoa a vela durante mucho tiempo, pasé mucho tiempo vendiendo en Vigia, São Caetano, Marapanim, Maracana, fuimos a Guamá, mi padre hacía esto. Después surgió la oportunidad de comprar un barco a motor, lo cual nos permitía ir para el Amazonas, y a partir de aquí la cosa mejoró un poco. Porque en Amazonas, veníamos para Belém a comprar los artículos de primera necesidad: azúcar, café, frijoles, arroz, galletas, queroseno, en fin. Llegabamos a Igarapé-Miri y abastecíamos con nuestra cachaza y desde ahí subíamos [el río] agarrando a algunos con nuestros productos (Entrevista de Oscar Rodrigues para el Programa "Papo no Tucupi" [https:// www.youtube.com/watch?v=53TUSE45iNg]).

Es esa red comercial que opera juntamente con las formas tradicionales de producción de bienes, en el sentido de garantizar el abastecimiento de las ciudades la que es paulatinamente desarticulada por el incremento de la urbanización y por las transformaciones en la sociedad, surgidas en virtud del desarrollo del proyecto de integración, que tienen la formación de la estructura metropolitana en Belém como un elemento de dichas transformaciones.

En ese sentido, se daban las condiciones materiales para que esos emprendedores actuasen cada vez más en la adaptación de sus negocios al nuevo escenario de la economía urbana y regional, esta vez operando de forma más sedentaria en Belém. Por un lado, las redes de conexión viales que posibilitaban la llegada de mercancías más económicas de otras regiones de Brasil y, a la vez, la formación de una economía metropolitana que sería la principal consumidora de esos mismos productos.

Por otra parte, existe un cambio en el alcance y en las dinámicas internas de los negocios, según lo descrito en otra declaración del empresario Oscar Rodrigues:

Después las cosas crecieron y mi padre logró comprar un barco más, luego otro barco y sentimos la necesidad de tener una parada en Belém (...) y nos quedábamos tratando esos negocios, comprando la mercancía de nuevo. Ya en [19]72, tenía condiciones económicas y compramos un edificio que tenía una tostadora de café (Entrevista de Oscar Rodrigues para el Programa "Papo no Tucupi" [https:// www.youtube.com/watch?v=53TUSE45iNg]).

Por consiguiente, el primer supermercado de la futura cadena es fundado en 1975, ubicado en los alrededores de la zona central de Belém, cerca de las conexiones con el comercio viabilizado por la navegación fluvial, pero ya incluido y siendo elemento de otra lógica de organización de estructuración del espacio urbano y regional, que se afianza durante las siguientes décadas.

\section{Expansión de la cadena de supermercados "Líder" y la dispersión metropolitana en Belém}

La lógica de expansión urbana y dispersión metropolitana que reestructuró la producción del espacio en Belém es el contexto para entender las dinámicas de expansión del Grupo Líder, como muestra de la ampliación y de la importancia del sector comercial en el circuito superior de la economía urbana en Belém. De esta forma, cuando tenemos en cuenta los tres sectores económicos - primario, secundario y terciario - en la primera década del año 2000, se aprecia que la Belém metropolitana prevalece en la economía regional. 
En el sector de servicios, por ejemplo, de los 10 municipios más grandes en el valor añadido del sector para el Estado de Pará (2009-2010), Belém fue responsable del 34,09\% del producto, mientras Ananindeua está en la segunda posición, con el 6,63\%. Es decir, más del 40\% de los servicios del Estado de Pará son proporcionados por las dos ciudades más grandes del espacio metropolitano.

Por otra parte, en el sector industrial - descentralizado en actividades del Estado en virtud de los proyectos de minería industrial, hidroenergéticos y relacionados a los frentes de expansión del agronegocio - existe una producción poco relevante, teniendo en cuenta por los menos los dos municipios más importantes a nivel económico. Belém, respecto al total del valor agregado del sector industrial ocupa la segunda posición en el estado, con solamente un $8,59 \%$ de la producción industrial. Ananindeua ocupa la séptima posición, con el 2,50\% del PIB industrial estatal (Pará, 2012, p. 23).

En términos sectoriales, los datos del Instituto Brasileño de Geografía y Estadística (2010) indican que la economía de la RMB se compone en gran memdida de actividades terciarias a principios del año 2000, y que dichas actividades por sí mismas respondieron por el 53,21\% del PIB metropolitano en el año 2002. Esa participación económica sectorial significa una expresión aún más importante de la composición de la población económicamente activa de la metrópolis.

Teniendo en cuenta la participación relativa de la población económicamente activa en el sector con más importancia en la economía metropolitana, según la Tabla 5, se aclara la importancia progresiva del sector terciario, especialmente en los municipios que dieron origen a la RMB, Belém y Ananindeua, además del municipio de Castanhal, recientemente incorporado a la composición metropolitana y con fuerte dinamismo económico en ese sector:

Tabla 5. Región Metropolitana de Belém: evolución absoluta de la participación de la Población Económicamente Activa (PEA) en el sector terciario (1960 - 1995)

\begin{tabular}{|lrrrr|} 
& 1960 & 1970 & 1980 & 1995 \\
\hline Belém & 83.313 & 123.101 & 46.007 & 92.676 \\
\hline Ananindeua & 1.348 & 2.624 & 1.702 & 8.294 \\
\hline Castanhal & - & - & 2.015 & 3.803 \\
\hline RMB & - & 50.915 & 101.508 \\
\hline
\end{tabular}

Fuente: Codem (1978); Ipeadata (2019)

Además, el perfil sectorial de la economía metropolitana en Belém también puede ser evaluado por la distribución del personal ocupado. Según los datos de la Pesquisa Nacional por Amostra de Domicilio (PNAD) para el año 2003 , el $69,69 \%$, o más de $2 / 3$ del total de las personas ocupadas se concentraban en el sector terciario. En sintonía con esto, el sector secundario era responsable de absorver al $20,3 \%$ del personal ocupado, suponiendo, por lo tanto, el sector primario solamente un $1,35 \%$ residual.

Y es justamente dentro de esa realidad económica en la que se produce la lógica de dispersión del espacio en Belém y la formación de la Región Metropolitana, como una muestra institucional de procedimientos de restauración territorial, y que la red de supermercados "Líder" acompañará como parte de sus estrategias comerciales en el espacio metropolitano.

El Gráfico 1 refleja la relevancia del ramo de supermercados en el territorio paraense: este sector es responsable del $5 \%$ de la totalidad del sector a nivel nacional, a pesar de que la población paraense corresponda, en comparación, al 3,98 \% del total de la población nacional (Tabla 6). 
En lo que a distribución regional de la renta nacional se refiere, la media de renta paraense es el equivalente a poco menos de la mitad de la renta nacional per cápita, según lo expuesto en la Tabla 6 . Lo cual es una demostración de la fuerza relativa de esas empresas, pues incluso actuando en una región de renta media muy inferior a la renta media nacional, logra alcanzar una expresión económica en el escenario brasileño.

Entonces, se puede demostrar que la participación de estos grupos en la economía regional no está solo restringida a la economía metropolitana de Belém y a su círculo inmediato, sino que tales cadenas, partiendo de sus tiendas por departamento (electrodomésticos, electrónicos y revistas), tienen una importancia relativa amplificada y que, aunque están básicamente controladas por empresas pertenecientes a grupos familiares en buena parte originados en la propia ciudad, como el mismo Grupo Líder, actualmente han conquistado relevancia en el escenario nacional, según se recoge en la Tabla 7 de la participación de algunos de estos grupos en la economía nacional.

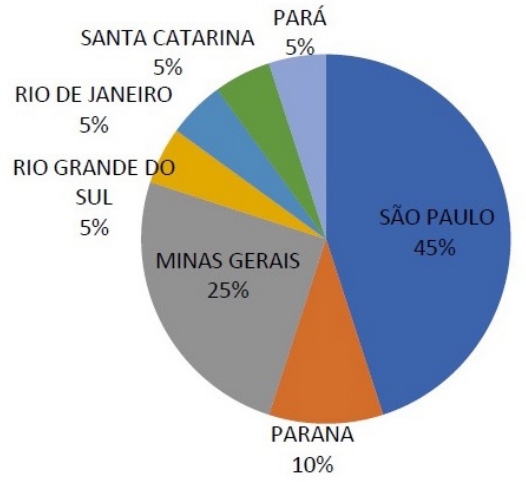

Gráfico 1. Brasil. concentración de empresas líderes del sector (2018). Fuente: Santos (2017).

Esto corrobora la interpretación de Santos (2004) sobre la existencia de una cantidad de personas con sueldos muy bajos o viviendo de actividades ocasionales, lo cual configura, dentro de la sociedad urbana, una separación entre aquellas personas para quienes es posible el acceso permanente a los bienes y servicios y aquellas que, teniendo idénticas necesidades, no cuentan con recursos para satisfacerlas. Simultáneamente, esto desemboca en una desigualdad, tanto cualitativa como cuantitativa, en el consumo. Estas diferencias son la causa y efecto de la existencia (creación y manutención) de dos circuitos paralelos de producción, distribución y consumo de bienes y servicios en esas ciudades.

Tabla 6. Comparación entre población brasileña y población paraense

\begin{tabular}{|c|c|c|c|c|c|}
\hline $\begin{array}{l}\text { POBLACIÓN } \\
\text { BRASILEÑA } \\
(2010)\end{array}$ & $\begin{array}{l}\text { POBLACIÓN } \\
\text { PARAENSE } \\
(2010)\end{array}$ & $\begin{array}{l}\text { POB. PARÁ / } \\
\text { POB. BRAS. } \\
\text { (\%) }\end{array}$ & $\begin{array}{l}\text { PIB PER } \\
\text { CÁPITA - } \\
\text { BRASIL (2008) }\end{array}$ & $\begin{array}{l}\text { PIB PER } \\
\text { CÁPITA - PARÁ } \\
\text { (2008) }\end{array}$ & $\begin{array}{l}\text { PIB PER } \\
\text { CÁPITA PARÁ } \\
\text { / PIB PER } \\
\text { CAPITA BRASIL }\end{array}$ \\
\hline 190.732.694 & 7.588.078 & 3,98 & $R \$ 15.990,00$ & $\mathrm{R} \$ 7.993,00$ & $0,499 \%$ \\
\hline
\end{tabular}

Fuente: IBGE (2010).

Tabla 7. Belém: Ranking de los supermercados (2018)

\begin{tabular}{|c|c|c|c|}
\hline $\begin{array}{l}\text { RANKING EN EL PAÍS } \\
\text { (BRASIL) }\end{array}$ & EMPRESA & $\mathbf{N}^{\circ}$ TIENDAS & $\mathbf{N}^{\circ}$ DE EMPLEADOS \\
\hline 12 & $\begin{array}{l}\text { Líder Comércio e Industria } \\
\text { LTDA }\end{array}$ & 23 & 12.365 \\
\hline 40 & Formosa Supermercados & 4 & 3.446 \\
\hline 49 & $\begin{array}{l}\text { Nazaré Companhia de } \\
\text { alimentos e Magazine LTDA }\end{array}$ & 5 & 1.955 \\
\hline 307 & Supermercado Cidade LTDA & 5 & 354 \\
\hline 539 & $\begin{array}{l}\text { Supermercado Estrela Dalva } \\
\text { Eireli }\end{array}$ & 1 & 58 \\
\hline
\end{tabular}

Fuente: Santos (2017). 
- ECONOMÍA URBANA Y DISPERSIÓN METROPOLITANA: EL ROL DE LAS CADENAS DE SUPERMERCADO

EN LA PRODUCCIÓN DEL ESPACIO METROPOLITANO EN BELÉM (PARÁ, BRASIL)

TIAGO VELOSO DOS SANTOS

Tabla 8. Grupo Líder: periodización de los supermercados en la Región Metropolitana de Belém

\begin{tabular}{|c|c|c|c|}
\hline $\begin{array}{l}\text { IDENTIFICACIÓN DEL } \\
\text { EMPRENDIMIENTO }\end{array}$ & $\begin{array}{l}\text { AÑO DE } \\
\text { INAUGURACIÓN }\end{array}$ & $\begin{array}{l}\text { MUNICIPIO } \\
\text { DE LA RMB }\end{array}$ & $\begin{array}{l}\text { UBICACIÓN EN LA ESTRUCTURA } \\
\text { METROPOLITANA }\end{array}$ \\
\hline Líder Condor & 1975 & Belém & $\begin{array}{l}\text { Barrio en los alrededores del núcleo } \\
\text { metropolitano }\end{array}$ \\
\hline Líder Alcindo Cacela & 1981 & Belém & Barrio del núcleo metropolitano \\
\hline Líder Doca & 1986 & Belém & Barrio del núcleo metropolitano \\
\hline Líder Cidade Velha & 1991 & Belém & Barrio del núcleo metropolitano \\
\hline Líder Castanheira & 1993 & Belém & Área de expansión metropolitana \\
\hline Líder Praça Brasil & 1995 & Belém & Barrio en los alrededores del núcleo \\
\hline Líder Batista Campos & 1995 & Belém & Barrio del núcleo metropolitano \\
\hline Líder Humaitá & 1995 & Belém & $\begin{array}{l}\text { Barrio en los alrededores del núcleo } \\
\text { metropolitano }\end{array}$ \\
\hline Líder Icoaraci & 1995 & Belém & Área de expansión metropolitana \\
\hline Líder BR & 1995 & Ananindeua & Área de expansión metropolitana \\
\hline Líder Castanhal & 1996 & Castanhal & Área de expansión metropolitana \\
\hline Líder Pedreira & 2007 & Belém & Barrio en los alrededores del núcleo \\
\hline Líder Independência & 2009 & Ananindeua & Área de expansión metropolitana \\
\hline Líder Canudos & 2010 & Belém & Barrio en los alrededores del núcleo \\
\hline Líder Cidade Nova & 2012 & Ananindeua & Área de expansión metropolitana \\
\hline Líder Barcarena & 2013 & & Área de expansión metropolitana \\
\hline Líder Augusto Montenegro & 2015 & Ananindeua & Área de expansión metropolitana \\
\hline Líder Marambaia & 2016 & Belém & Área de expansión metropolitana \\
\hline
\end{tabular}

Fuente: El autor

Si se ve desde una perspectiva histórica y geográfica, particularmente el Grupo Líder ${ }^{11}$ ha evolucionado de forma muy asociada a la progresiva expansión del espacio metropolitano de Belém. Desde dicha perspectiva, la Tabla 8 presenta la consistente apertura de los emprendimientos del grupo asociándola a la ubicación en el interior de la estructura metropolitana de Belém.

El Mapa 2 muestra la relación de la ubicación de los emprendimientos comerciales del Grupo Líder con la actual estructura metropolitana de Belém. En este sentido, es menester recordar que la Región Metropolina de Belém pasó por la expansión del tejido urbano teniendo como consecuencia la incorporación de nuevos municipios. Si este proceso se compara con la expansión de los emprendimientos del Grupo Líder, es clara su coexistencia en espacio y tiempo.

La periódica expansión de los emprendimientos del Grupo demuestra similitud espaciotemporal con el proceso de dispersión metropolitana en Belém. En un principio, los emprendimientos estaban ubicados en urbanizaciones del área central de Belém, el núcleo de actividades metropolitanas o el círculo inmediato de ese núcleo de la metrópolis. Esto sucedió entre las décadas de 1970 y 1980, cuando se inauguraron las primeras tiendas y se abrieron la segunda y tercera sucursal de la cadena de supermercados. 
Desde la década de 1990, esta configuración de distribución espacial sufre cambios producto de la apertura de tres sucursales del Grupo: la primera, "Líder Icoaraci”, ubicada en el Distrito del mismo nombre y que forma parte del municipio de Belém; sin embargo, este distrito queda alejado del área central, formando parte del área de expansión urbana y metropolitana. La segunda sucursal es "Líder BR", en el municipio de Ananindeua. Esta sucursal se localiza en el kilómetro 05 de la carretera federal BR-316 y configura un segundo vector de expansión metropolitana de Belém. La tercera sucursal es "Líder Castanhal", en el municipio homónimo y que vendría a transformarse en municipio constitutivo de la RMB en el año 2011. La elección de Castanhal no fue accidental, pues este municipio pertenece al grupo de localidades que se destacan en términos económicos y de inversiones en infraestructura a través de la cooperación con el gobierno estatal.

La expansión de las sucursales continúa hasta hoy en día, provocando que en la Región Metropolitana de Belém el Grupo Líder ostente la mayor cantidad de supermercados distribuidos por los municipios más representativos, económica y demográficamente hablando, de la dinámica interna de la metrópolis, con una expectativa de inauguración de por lo menos una unidad más en la zona central de Belém.

Es sumamente relevante el hecho de que, entre esas 18 sucursales del Grupo, cinco hayan sido inauguradas en el período 2007-2013, justo el de mayor crecimiento de la economía brasileña en las décadas más recientes, y que otras dos hayan sido inauguradas en los años siguientes, 2015 y 2016, estando ya en recesión, pero siendo efectivamente planificadas en el período anterior. Es evidente que, desde el punto de vista de estructura metropolitana, la distribución de las sucursales obedece a un patrón que sigue los vectores de expansión urbana: la BR-316 en dirección a los municipios de la RMB y la Avenida Augusto Montenegro, en la cual se encuentra la principal área de expansión de la frontera urbano-inmobiliaria en Belém. Sin embargo, existe una significativa concentración de sucursales en el núcleo metropolitano, ratificando la manutención de la relevancia del área central de Belém, vinculada a la centralización de los consumidores.

\section{Conclusión}

Durante la formación de la Región Metropolitana de Belém, fue analizada la labor de uno de los agentes productores de espacio urbano con mayor impacto en la economía de la metrópolis, es decir las cadenas de supermercados minoristas, con especial énfasis en el Grupo Líder. El análisis de la producción de espacio, de acuerdo con la perspectiva de la reorganización económica de la formación metropolitana de Belém, condujo a la identificación de lógicas de operación de las cadenas de supermercados del Grupo en paralelo a la expansión del tejido metropolitano del cual Belém es el núcleo. 
Las evidencias que se mostraron a lo largo del texto, tales como la cartografía temática, producidas a partir de la revisión documental y de las observaciones sistemáticas de campo, pretendieron demostrar que el surgimiento de esos grupos minoristas se produjo a partir del fortalecimiento del sector terciario de la economía urbana en Belém, especialmente partiendo de la modernización de alguno de los primeros locales, y en el mismo periodo de formación del tejido metropolitano, siendo entonces expresión de una economía que pasó a ser hegemónicamente organizada alrededor del sector terciario moderno.

Así, el ambiente económico de la Belém metropolitana en formación a partir de los años 1960 es producto, condición y medio de un contexto más grande de las políticas de desarrollo e integración nacional que, de manera indirecta, propiciaron la desestructuración del pequeño sector industrial regional existente en la ciudad hasta entontes; además de provocar, paralelamente, la reestructuración de las actividades comerciales tradicionales, incapaces de competir con productos industrializados que ahora llegaban por la nueva conexión vial y que necesitaban modernizarse o corrían el riesgo de desaparecer, debido a la fundación de un mercado más competitivo, que fue exactamente el caso de nuestra referencia empírica de análisis: el Grupo Líder.

Más allá de ese período inicial de formación de la economía metropolitana en Belém, la dinámica de dispersión del tejido urbano en las siguientes décadas, de 1980 a 2010, estuvo conectada a las estrategias de expansión de los emprendimientos comerciales que progresivamente continuaron modernizándose. De este modo, además de estos elementos inductores de dicha expansión urbana y metropolitana, un posible indicador económico siguió las tendencias de dispersión de la metrópolis, fortaleciendo la multiplicación las cadenas de supermercados como vectores de oferta laboral y actividades en una economía metropolitana que, desde entonces, se volvía más dependiente del sector terciario. 


\section{NOTAS}

1 Para Lencioni (2008), la metropolización del espacio hace referencia a la difusión de la urbanización por la sociedad y por el territorio, intensificada al punto de engendrar la forma contemporánea de urbanización en las sociedades de mercado avanzadas, la metrópolis. La calidad metropolitana en el espacio urbano actual es entendida partiendo de conceptos de concentración y de centralización del capital, y expresa un tipo de metamorfosis socio-espacial, que constituye la metropolización del espacio (Lencioni, 2008, p. 52).

2 Para Santos \& Silveira (2001), la urbanización del territorio y de la sociedad caracteriza a Brasil al inicio del siglo XXI. Dicho proceso es entendido como diseminación desigual del medio técnico-científicoinformacional.

${ }^{3}$ Teniendo en cuenta los siete municipios que componen la Región Metropolitana de Belém, la población total de la zona metropolitana es de 2.422.481 millones de personas, siendo la segunda mayor zona metropolitana regional, detrás solamente de la Región Metropolitana de Manaus, con 13 municipios y un total de 2.676.936 millones de habitantes (IBGE, 2010).

${ }^{4}$ Creada en 1973, la Región Metropolitana de Belém estaba constituida por los municipios de Belém y Ananindeua. Luego, en la década de 1990, fue ampliada con la incorporación de los municipios de Benevides, Santa Bárbara do Pará y Marituba. En 2009, el municipio Santa Izabel do Pará pasa a formar parte de la RMB y, en 2011, el municipio de Castanhal, configurando la actual Región Metropolitana de Belém con siete municipios.

${ }^{5}$ Estadísticas del PIB Municipal a precios del año 2000 (R\$). Para 1999-2007: Sistema de Cuentas Regionales. Concepto utilizado a partir de 1999 a precios básicos. Para los años de censos de 1970 a 1996, elaborado por el IPEA. Para datos anteriores a 1999, la estimación del PIB de las actividades a nivel municipal no son consistentes con las del nivel estatal y nacional. El problema se debe a la utilización de metodologías distintas. Deflactor Implícito del PIB nacional (Ipeadata).

${ }^{6}$ Reflejo de estas formas tradicionales de actividad económica, es la práctica del pastoreo en Belém, constituyendo las llamadas "vaquerías", que surtían a la ciudad de leche in natura (Trindade Jr, 1993). O la producción de caña de azúcar, cachaza y palmitos en Abaetetuba (Anderson, 1991). Ambas actividades, vinculadas a un estilo de vida rural, pero también relacionadas al consumo urbano, experimentaron un declive después de las modernizaciones técnicas y la llegada de la carretera.

7 Hasta la década de 1950, Belém contaba con un parque industrial que satisfacía parcialmente la demanda local y regional, destacando dos tipos de industrias: la producción de materias primas semi-manufacturadas utilizada para la exportación (caucho laminado, cuero curtido, madera, aceites y esencias, castañas, prensado de yuta, selección de semillas oleaginosas) y la producción de bienes de consumo centrados en las necesidades locales y alimentos (alimentos, bebidas, productos de higiene y limpieza, calzado, sombreros, ropa y construcción civil) (Mourão, 2017).

8 Se puede hablar de un circuito superior formado por bancos, comercio e industria de exportación, industria urbana moderna, servicios modernos, mayoristas y transportistas. El circuito inferior consiste en formas de fabricación (no "capital intensivo"), por los servicios "al detal" y por el comercio no moderno y de pequeño porte. En el circuito superior, se pueden distinguir las actividades "puras" y "mezcladas". La industria urbana moderna, el comercio y los servicios modernos son los elementos "puros", ya que son actividades específicas de la ciudad y del circuito superior, simultáneamente (Santos, 2004, pp. 40-41).

9 El grupo Y.Yamada poseía hasta el año 2015 dos sucursales en la ciudad de Macapá, capital del estado Amapá.

10 Banco de Amazônia S.A (BASA), la Superintendência de Desarrollo da Amazônia (Sudam), Eletronorte, la Companhia de Produções de Recursos Minerais (CPRM), todas con sede en Belém hasta hoy.

11 En la actualidad (2019), el Grupo Líder es un conjunto de emprendimientos minoristas en distintos sectores que incluye, además de las cadenas de supermercados "Líder", la cadena "Magazan" (tienda por departamento), con 15 tiendas; Farmalider (medicamentos al por menor), con 21 tiendas; las Óticas Líder, con 18 tiendas; el Cartão Liderzan para crédito al consumidor; la mara "Café Líder". Adicionalmente, es el grupo encargado de administrar "Castanheira" Shopping Center; posee Fomento Mercantil en Castanheira Empreendimentos y la hacienda "Tres Marias", propiedad de la familia fundadora. La facturación total del Grupo para el año fiscal de 2015 fue de 1.987 mil millones de reales, ocupando el puesto 46 de las mayores cadenas minoristas del país (Revista Exame, 2015). 


\section{REFERENCIAS BIBLIOGRÁFICAS}

Anderson, S. D. (1991). Engenhos na várzea: uma análise do declínio de um sistema de produção tradicional na Amazônia. In P. Léna, \& A. E. Oliveira. Amazônia: a fronteira agrícola 20 anos depois (pp. 101-121). Belém: Museu Emílio Goeldi. Recuperado de [http://repositorio. museu-goeldi.br:8080/jspui/handle/mgoeldi/397].

Browder, J. O., \& Godfrey, B. J. (2006). Cidades da floresta: urbanização, desenvolvimento e globalização na Amazônia brasileira. Manaus: Editora da Universidade do Estado do Amazonas.

Codem. (1978). Análise das funções metropolitanas. Belém: Companhia de Desenvolvimento da Área Metropolitana de Belém.

Conexão Belém do Pará [Conexão Belém do Pará]. (22 de agosto de 2017). Entrevista com Oscar Rodrigues, "Papo no Tucupi". Recuperado de [https://www.youtube. $\mathrm{com} /$ watch? $\mathrm{v}=53$ TUSE45iNg].

Conselho Nacional De Economia (1962). Exposição geral da situação econômica do Brasil. Brasília: Conselho Nacional de Economia.

Corrêa, R. L. (1989). O espaço urbano. São Paulo: Ática.

Instituto Brasileiro de Geografia e Estatística (IBGE). (2010). Informações dos Censos demográficos brasileiros: 1950, 1960, 1970, 1980, 1991, 2000, 2010. Brasília. Recuperado de [https://seriesestatisticas.ibge. gov.br/].

Instituto de Pesquisa Econômica Aplicada (IPEA). (2015). Governança metropolitana no Brasil. caracterização e quadros de análise comparativa da governança metropolitana no Brasil. Rio de Janeiro: Instituto de Pesquisa Econômica Aplicada. Recuperado de [https:// www.ipea.gov.br/portal/index.php?option=com_content $\& v i e w=$ article\&id $=26587 \&$ Itemid $=1]$.

Ipeadata. (2019). Sistema de dados. Brasília: Instituto de Pesquisa Econômica Aplicada 2019. Recuperado de [http://www.ipeadata.gov.br/Default.aspx].

Lefébvre, H. (1991) O direito à cidade. São Paulo: Contexto.

Lencioni, S. (2008). Concentração e centralização das atividades urbanas: uma perspectiva multiescalar. reflexões a partir do caso de São Paulo. Revista de Geografia Norte Grande, n 39, 07-20.

Moreira, E. (1989). Belém e sua expressão geográfica. Pará. Obras reunidas de Eidorfe Moreira. Belém: Cejup.

Mourão, L. (2017). Memórias da Indústria paraense. XII Congresso Brasileiro de História Econômica \& XIII Conferência Internacional de História das Empresas (127).
Pará. (2012). Diretrizes de ordenamento territorial da Região Metropolitana de Belém. Belém: Governo do Estado do Pará.

Revista Exame. (2015). Recuperado de [https://exame. abril.com.br/negocios/as-50-maiores-varejistas-dobrasil-em-faturamento-em-2015/]. Consultado em [2403-2020].

Queiróz dos Santos, F. (2019). Produção do espaço e expansão urbana na Região Metropolitana de Belém: a chegada do empreendimento Líder no conjunto Cidade Nova em Ananindeua. Monografia de conclusão de curso, Instituto Federal de Educação, Ciência e Tecnologia do Pará, Belém, Pará, Brasil.

Santos, C. S. (2017). Informalidade do trabalho na Metrópole periférica: estudo crítico na economia brasileira e sua evolução na RMB entre as décadas de 1990 e 2010. Monografia de conclusão de curso, Universidade Federal do Pará, Belém, Pará, Brasil.

Santos, M. (2004). O espaço dividido: os dois circuitos da economia urbana nos países subdesenvolvidos. São Paulo: Edusp.

Santos, M., \& Silveira. M. L. (2001). O Brasil: território e sociedade no início do século XXI. São Paulo: Record.

Santos, T. V. (2017). Metropolização regional e suburbanização condominial: aspectos da dinâmica da dispersão urbana na Região Metropolitana de Belém. Boletim Campineiro de Geografia, 7(1) 91-116.

Trindade Júnior, S. C. C. (1997). Produção do espaço e uso do solo urbano em Belém. Belém: Editora da Universidade Federal do Pará.

Trindade Júnior, S. C. C. (1998). A cidade dispersa: os novos espaços de assentamentos em Belém e a reestruturação metropolitana. Tese de doutorado, Universidade de São Paulo, São Paulo, Brasil.

Trindade Júnior, S. C. C. (2000). A natureza da urbanização na Amazônia e sua expressão metropolitana. Revista Geografares, 1(1). 117-130.

Trindade Júnior, S. C. C. (2006). Assentamentos urbanos e metropolização na Amazônia brasileira: o caso de Belém. Revista GEOUSP, Espaço \& Tempo, 2(2). 39-52.

Trindade Júnior, S. C. C., \& Leite, G. C. S. (2019). Metrópole e economia urbana na Amazônia: Olhando Belém na perspectiva da Teoria dos Circuitos. Revista e-metropolis, 36(10), 9-18.

Tupiassu, A., \& Santos, R. (1967). Introdução ao Estudo Sociológico e Econômico do Estado do Pará. Belém: Governo do Estado do Pará.

Vicentini, Y. (2004). Cidade e história na Amazônia. Curitiba: UFPR. 